\title{
B2045+265: A NEW FOUR-IMAGE GRAVITATIONAL LENS FROM CLASS ${ }^{1}$
}

\author{
C. D. Fassnacht, R. D. Blandford, ${ }^{2}$ J. G. Cohen, K. Matthews, T. J. Pearson, \\ A. C. S. READHEAD, AND D. S. WoMbLE ${ }^{3}$ \\ Palomar Observatory, 105-24, California Institute of Technology, Pasadena, CA 91125 \\ S. T. MYERS \\ Department of Physics and Astronomy, University of Pennsylvania, 209 South 33d Street, Philadelphia, PA 19104-6396 \\ I. W. A. Browne, N. J. JaCKson, D. R. Marlow, AND P. N. Wilkinson \\ Nuffield Radio Astronomy Laboratories, Jodrell Bank, University of Manchester, Macclesfield, Cheshire SK11 9DL, England, UK \\ L. V. E. KoOpMans \\ Kapteyn Astronomical Institute, Postbus 800, NL-9700 AV Groningen, Netherlands
}

A. G. DE BRUYN ${ }^{4}$ AND R. T. SCHILIZZI

Netherlands Foundation for Research in Astronomy, Postbus 2, NL-7990 AA Dwingeloo, Netherlands

AND

M. BREMER AND G. MiLEY

Leiden Observatory, Postbus 9513, NL-2300 RA Leiden, Netherlands

Received 1998 May 18; accepted 1998 October 29

\begin{abstract}
We have discovered a new gravitational lens in the Cosmic Lens All-Sky Survey (CLASS). The lens B2045 +265 is a four-image system with a maximum separation of 1"9. A fifth radio component is detected, but its radio spectrum and its positional coincidence with infrared emission from the lensing galaxy strongly suggest that it is the radio core of the lensing galaxy. This implies that the B2045+265 lens system consists of a flat-spectrum radio source that is being lensed by another flat-spectrum radio source. Infrared images taken with the Hubble Space Telescope and the Keck I Telescope detect the lensed images of the background source and the lensing galaxy. The lensed images have relative positions and flux densities that are consistent with those seen at radio wavelengths. The lensing galaxy has magnitudes of $J=19.2, m_{\mathrm{F} 160 \mathrm{w}}=18.8$, and $K=17.6 \mathrm{mag}$ in a 1".9 diameter aperture, which corresponds to the size of the Einstein ring of the lens. Spectra of the system taken with the Keck I Telescope reveal a lens redshift of $z_{l}=0.8673$ and a source redshift of $z_{s}=1.28$. The lens spectrum is typical of an Sa galaxy. The image splitting and system redshifts imply that the projected mass inside the Einstein radius of the lensing galaxy is $M_{\mathrm{E}}=4.7 \times 10^{11} h^{-1} M_{\odot}$. An estimate of the light emitted inside the Einstein radius from the $K$ magnitude gives a mass-to-light ratio in the rest-frame $B$ band of $\left(M / L_{B}\right)_{\mathrm{E}}=$ $20 h\left(M / L_{B}\right)_{\odot}$. Both the mass and mass-to-light ratio are higher than what is seen in nearby Sa galaxies. In fact, the implied rotation velocity for the lensing galaxy is $2-3$ times higher than what is seen in nearby spiral galaxies. The large projected mass inside the Einstein ring radius may be the result of a significant amount of dark matter in the system, perhaps from a compact group of galaxies associated with the primary lensing galaxy; however, it may also arise from a misidentification of the source redshift. A simple model of the gravitational potential of the lens reproduces the image positions well, but further modeling is required to satisfy the constraints from the image flux density ratios. With further observations and modeling, this lens may yield an estimate of $H_{0}$.
\end{abstract}

Key words: distance scale - galaxies: distances and redshifts - gravitational lensing -

quasars: individual (B2045+265)

\footnotetext{
${ }^{1}$ Based on observations made with the National Radio Astronomy Observatory, which is operated by Associated Universities, Inc., under cooperative agreement with the National Science Foundation; with the NASA/ESA Hubble Space Telescope, obtained at the Space Telescope Science Institute which is operated by AURA, Inc., under NASA contract NAS 5-26555; and with the W. M. Keck Observatory, which is operated as a scientific partnership among the California Institute of Technology, the University of California, and the National Aeronautics and Space Administration. The Keck Observatory was made possible by the generous financial support of the W. M. Keck Foundation.

${ }^{2}$ Also Theoretical Astrophysics, 130-33, California Institute of Technology, Pasadena, CA 91125.

${ }^{3}$ Current address: Monterey Institute for Research in Astronomy, 200 Eighth Street, Marina, CA 93955; dsw@mira.org.

${ }_{4}^{4}$ Also Kapteyn Astronomical Institute, Postbus 800, NL-9700 AV Groningen, Netherlands.
}

\section{INTRODUCTION}

The Cosmic Lens All-Sky Survey (CLASS; Myers et al. 1999 ) is a large-scale survey for gravitational lenses among flat-spectrum radio sources. The primary goals of CLASS are to find lenses that may be suitable for determinations of the Hubble constant, $H_{0}$ (see, e.g., Refsdal 1964; Blandford \& Narayan 1992), and to study the lensing rate in a large, homogeneous sample in order to place limits on the cosmological constant, $\Lambda$ (see, e.g., Turner, Ostriker, \& Gott 1984; Turner 1990; Fukugita, Futamase, \& Kasai 1990; Fukugita \& Turner 1991). Over 12,000 sources have been observed with the Very Large Array (VLA) in three sessions - the first in the spring of 1994 (CLASS 1; 3300 sources), the second 
in the summer of 1995 (CLASS 2; 4500 sources), and the third in the spring of 1998 (CLASS $3 ; \sim 5000$ sources). The vast majority of flat-spectrum radio sources are dominated by emission from a single compact core; all CLASS sources containing multiple compact components are selected as gravitational lens candidates, amounting to 50-100 candidates in each phase of the survey. These are followed up with high-resolution radio imaging using MERLIN; the best surviving candidates are then imaged using the Very Long Baseline Array (VLBA). During this procedure, the majority of VLA candidates are rejected based on surface brightness and morphology criteria. The candidates that survive the radio filters are then investigated further with optical and/or infrared imaging, spectroscopy, and more radio imaging. By these means we have discovered 11 new lenses in the survey in addition to the one reported here. We are also investigating an additional $\sim 25$ promising lens candidates.

We use $H_{0}=100 h \mathrm{~km} \mathrm{~s}^{-1} \mathrm{Mpc}^{-1}$ and, except where noted, assume $q_{0}=0.5$ throughout this paper.

\section{VLA OBSERVATIONS}

The lens system B2045+265 (GB6 J2047+2643) was observed on 1995 September 2 as part of CLASS 2. The observations were made at $8.4 \mathrm{GHz}$ with the VLA in the A configuration, giving a resolution of $\sim 0$ ".25. Observation and data reduction techniques for the survey will be discussed in Myers et al. (1999). The survey image of B2045 + 265 shows four components, with a possible detection of a fifth, weak component. The system has a standard
TABLE 1

RADIO OBSERVATIONS

\begin{tabular}{llrcl}
\hline \hline Array & \multicolumn{1}{c}{ Date } & \multicolumn{1}{c}{$\begin{array}{c}v \\
(\mathrm{GHz})\end{array}$} & $\begin{array}{c}t_{\text {tot }} \\
(\text { minutes })\end{array}$ & $\begin{array}{c}\text { Angular Resolution } \\
(\operatorname{arcsec})\end{array}$ \\
\hline VLA $\ldots \ldots \ldots \ldots$ & 1995 Sep 2 & 8.5 & 0.5 & 0.25 \\
VLA $\ldots \ldots \ldots \ldots$ & 1995 Sep 11 & 14.9 & 7 & 0.14 \\
VLBA $\ldots \ldots \ldots \ldots$ & 1995 Nov 12 & 5.0 & 35 & 0.0025 \\
VLA $\ldots \ldots \ldots \ldots$ & 1996 Dec 31 & 1.4 & 12 & 1.5 \\
VLA $\ldots \ldots \ldots \ldots$ & 1996 Dec 31 & 4.9 & 12 & 0.43 \\
VLA $\ldots \ldots \ldots \ldots$. & 1996 Dec 31 & 8.5 & 192 & 0.25 \\
VLA $\ldots \ldots \ldots \ldots$ & 1996 Dec 31 & 14.9 & 20 & 0.14 \\
MERLIN ....... & 1997 Nov 30 & 5.0 & 750 & 0.50 \\
\hline
\end{tabular}

TABLE 2

Component Positions

\begin{tabular}{lrrc}
\hline \hline Component & \multicolumn{1}{c}{$\Delta \alpha^{\mathrm{a}}$} & \multicolumn{1}{c}{$\Delta \delta^{\mathrm{a}}$} & \multicolumn{1}{c}{$\sigma_{x}^{\mathrm{b}}$} \\
\hline $\mathrm{A} \ldots \ldots \ldots \ldots$ & 0.000 & 0.000 & $\ldots$ \\
$\mathrm{B} \ldots \ldots \ldots \ldots$ & -0.134 & -0.248 & 0.001 \\
$\mathrm{C} \ldots \ldots \ldots \ldots$ & -0.288 & -0.789 & 0.001 \\
$\mathrm{D} \ldots \ldots \ldots \ldots$ & +1.628 & -1.007 & 0.006 \\
$\mathrm{E} \ldots \ldots \ldots \ldots$ & +1.121 & -0.824 & 0.005 \\
\hline
\end{tabular}

${ }^{\text {a }}$ Positions relative to component $\mathrm{A}$ are taken from the 1996 December 31, 8.5 GHz map. Component $\mathrm{A}$ is at $20^{\mathrm{h}} 47^{\mathrm{m}} 20^{\mathrm{s}} 29,+26^{\circ} 44^{\prime} 02^{\prime \prime} .7$ (J2000).

${ }^{b}$ Uncertainties in relative positions calculated using the 1996 December 31, 8.5 GHz data and assuming that the uncertainties in the component flux densities are 3 times the rms noise level in the map.

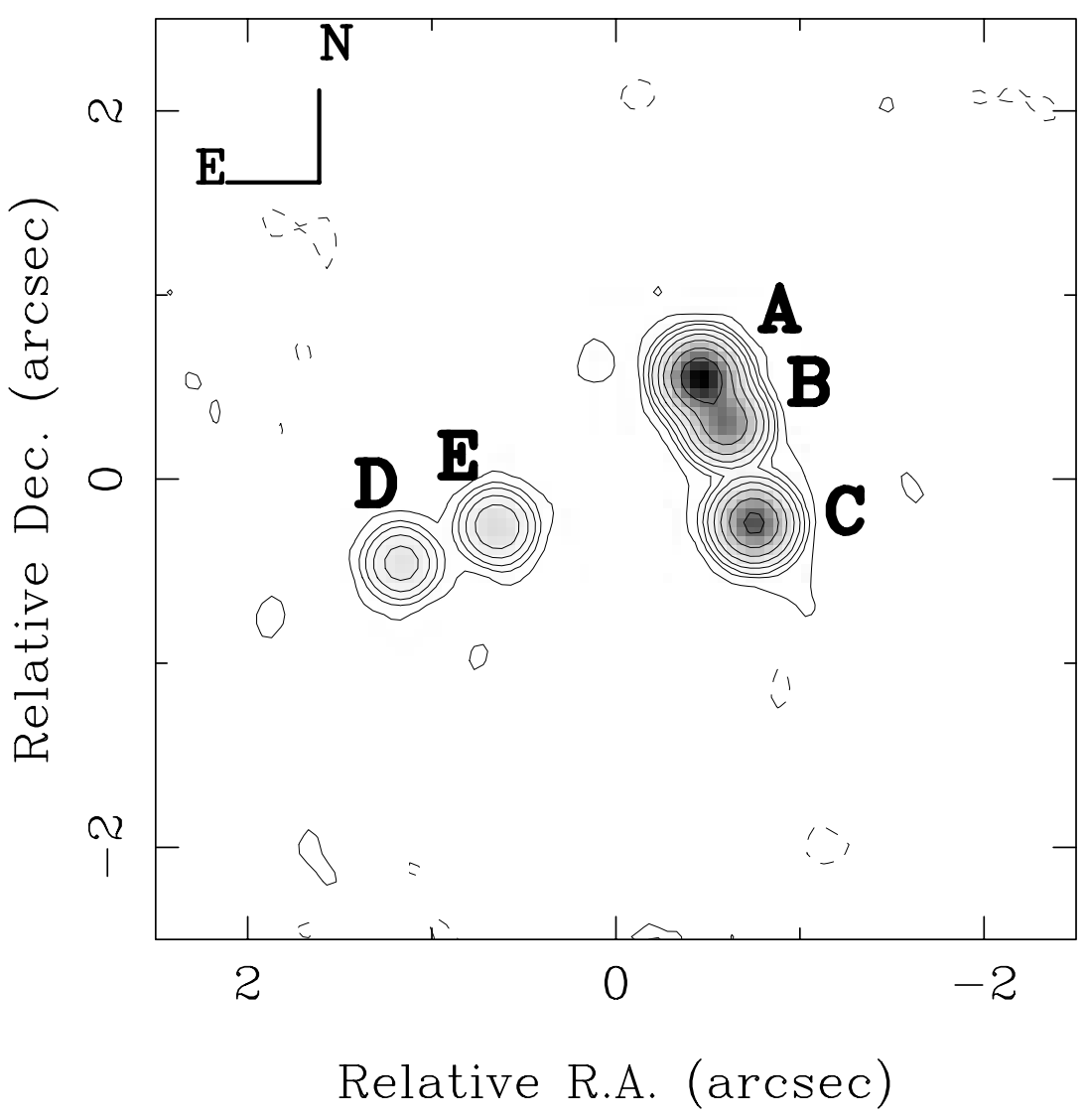

Fig. 1.-1996 December 8.5 GHz map. The contours are $(-2.5,2.5,5,10,20,40,80,160,320,640)$ times the rms noise level of $0.0145 \mathrm{mJy}^{\mathrm{beam}}{ }^{-1}$. Map made by fitting point-source components, with flux densities listed in Table 3, to the $(u, v)$ data, and restoring with a 0 ".27 $\times 0$ ".23 restoring beam. 


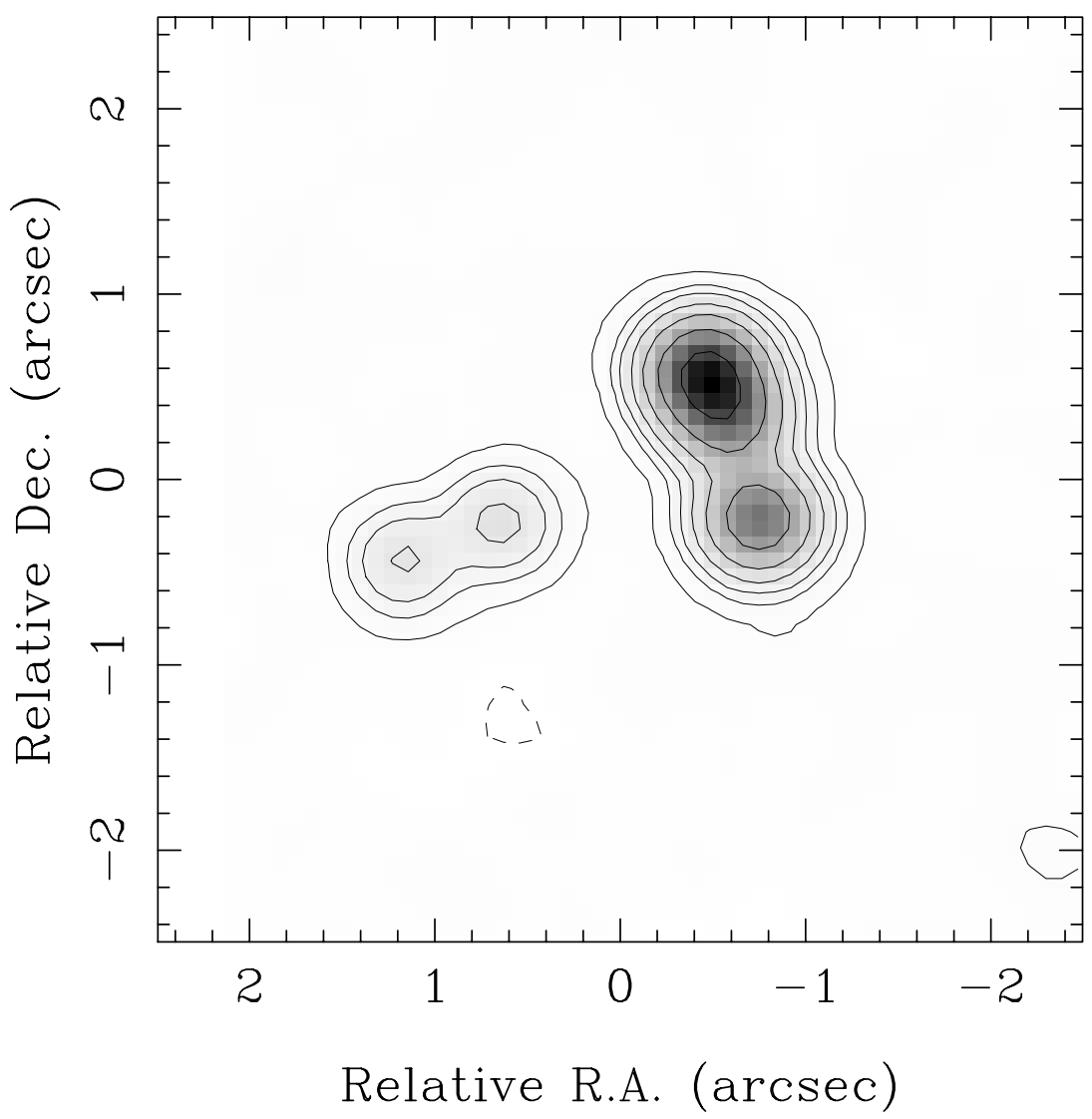

Fig. 2. - 1996 December $4.9 \mathrm{GHz}$ map. The contours are $(-2.5,2.5,5,10,20,40,80,160,320)$ times the rms noise level of $0.0621 \mathrm{mJy} \mathrm{beam}^{-1}$. Map made by fitting point-source components, with flux densities listed in Table 3, to the $(u, v)$ data, and restoring with a $0.44 \times 0$ ".41 restoring beam.

lens geometry (e.g., Blandford \& Narayan 1992) and is similar in appearance to B1422 + 231 (Patnaik et al. 1992).

The source was reobserved with the VLA on 1996 December 31. Observations were made in the A configuration at $1.4,4.9,8.5$, and $14.9 \mathrm{GHz}$. Details of the observations are given in Table 1 . The source 3C 286 was used as a flux calibrator. Phase calibrators were selected from the VLA calibrator list. The data were calibrated using standard AIPS routines, and maps were made using DIFMAP (Shepherd, Pearson, \& Taylor 1994). At the frequencies above $1.4 \mathrm{GHz}$, the source can be characterized as five point components, with no significant extended structure. Hence, the maps were made by fitting five point components to the data and then repeating a cycle of model fitting and phase self-calibration. The self-calibration timescale was set to the length of the individual scans at each frequency. Both the positions and the flux densities were allowed to vary in the model fitting. At $1.4 \mathrm{GHz}$ the beam size is large compared with the component separation, so the model fitting was done by fixing the component positions at their $8.5 \mathrm{GHz}$ values and varying only the component flux densities. In addition, there were confusing sources in the $1.4 \mathrm{GHz}$ map that had to be included as components in the model.

The final maps are shown in Figures 1-4. In all cases, the morphology seen in the discovery map is duplicated, with five distinct components clearly present in the high dynamic range 5 and $8.5 \mathrm{GHz}$ maps. Component positions and flux densities were obtained using the model-fitting procedures in DIFMAP. The brightest component (A) has an $8.5 \mathrm{GHz}$ flux density of $16.55 \mathrm{mJy}$ and is located at $20^{\mathrm{h}} 47^{\mathrm{m}} 20.29$, $+26^{\circ} 44^{\prime} 02^{\prime \prime} 7$ (J2000); the positions of the other components relative to component A are given in Table 2 (see Fig. 1 for

TABLE 3

Component Flux Densities

\begin{tabular}{|c|c|c|c|c|c|c|c|c|}
\hline Date & Array & $\begin{array}{c}v \\
(\mathrm{GHz})\end{array}$ & $\begin{array}{c}S_{A} \\
(\mathrm{mJy})\end{array}$ & $\begin{array}{c}S_{B} \\
(\mathrm{mJy})\end{array}$ & $\begin{array}{c}S_{C} \\
(\mathrm{mJy})\end{array}$ & $\begin{array}{c}S_{D} \\
(\mathrm{mJy})\end{array}$ & $\begin{array}{c}S_{E} \\
\text { (mJy) }\end{array}$ & $\begin{array}{c}\mathrm{rms} \\
\left(\mathrm{mJy} \mathrm{beam}^{-1}\right)\end{array}$ \\
\hline 1995 Sep $2 \ldots \ldots \ldots$ & VLA & 8.5 & 18.4 & 9.42 & 14.8 & 2.41 & 1.83 & 0.28 \\
\hline 1995 Sep $11 \ldots \ldots$ & VLA & 14.9 & 15.5 & 9.18 & 11.8 & 1.22 & 2.36 & 0.32 \\
\hline 1995 Nov $12 \ldots \ldots$ & VLBA & 5.0 & 15.8 & 8.09 & 8.75 & $\ldots$ & $\ldots$ & 0.20 \\
\hline \multirow[t]{4}{*}{1996 Dec $31 \ldots \ldots$} & VLA & 1.4 & 29.02 & 15.73 & 19.92 & 2.81 & 2.18 & 0.08 \\
\hline & & 4.9 & 23.40 & 12.65 & 15.19 & 2.68 & 3.00 & 0.06 \\
\hline & & 8.5 & 16.55 & 8.90 & 10.99 & 1.77 & 2.34 & 0.01 \\
\hline & & 14.9 & 10.69 & 5.75 & 7.13 & 1.05 & 1.90 & 0.14 \\
\hline 1997 Nov $30 \ldots . .$. & MERLIN & 5.0 & 16.8 & 9.82 & 14.8 & 2.02 & 1.53 & 0.15 \\
\hline
\end{tabular}




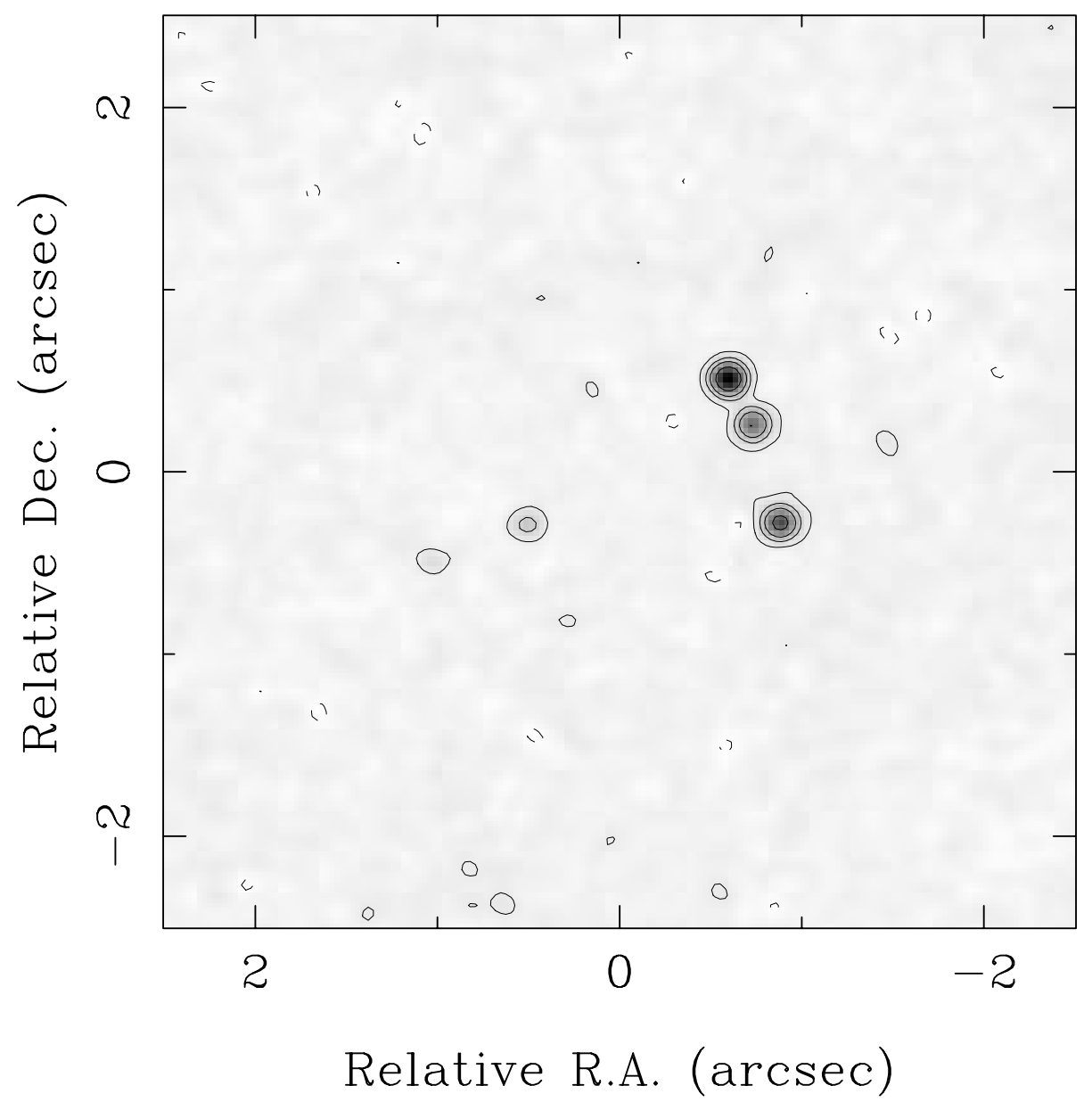

FIG. 3.-1996 December $15 \mathrm{GHz}$ map. The contours are $(-2.5,2.5,5,10,20,40)$ times the rms noise level of $0.139 \mathrm{mJy}^{\text {beam }}{ }^{-1}$. Map made by fitting point-source components, with flux densities listed in Table 3, to the $(u, v)$ data, and restoring with a 0 ".14 $\times 0$ ".13 restoring beam.

component labels). The positional uncertainties for the different components are estimated as the beam size at each frequency divided by the signal-to-noise ratio of the component flux densities; the positional errors in the $8.5 \mathrm{GHz}$ map are given in Table 2. The largest separation in the system is 1 ".9. At all frequencies, five point components are adequate to fit the data. No sign of extended emission is seen, even in the deep $8.5 \mathrm{GHz}$ map. The final component flux densities and rms noise levels for the maps are given in Table 3. In all cases the noise levels are within $5 \%$ of the thermal noise expected in the maps.

The $8.5 \mathrm{GHz}$ data were also analyzed to search for polarized emission. The polarization calibration was carried out in AIPS, using 3C 286 as the calibrator. The phase calibrator $2115+295$ was observed over a range of parallactic angles and was used to determine the instrumental polarization. The final maps are consistent with no polarized emission above $56 \mu \mathrm{Jy}^{\text {beam }}{ }^{-1}(0.34 \%$ of the peak unpolarized intensity).

Radio spectra for the five components are shown in Figure 5. Components $\mathrm{A}-\mathrm{C}$ have very similar spectra, with spectral indices of $\alpha_{1.4}^{4.9} \sim-0.2$ and $\alpha_{4.9}^{15} \sim-0.6\left(S_{v} \propto v^{\alpha}\right)$. The spectra of components $\mathrm{D}$ and $\mathrm{E}$ differ from those of the three brighter components. In order to determine which, if either, of these two components is the counterimage to components A, B, and C, flux density ratios (shown in Table 4) are computed for each component with respect to component A. By computing flux density ratios at each fre- quency, it is possible to avoid uncertainties due to errors in the absolute flux calibration. The resulting curves (Fig. 6), normalized to their values at $8.5 \mathrm{GHz}$, clearly indicate that the spectrum of component $\mathrm{E}$ differs from those of the other four components.

\section{MERLIN AND VLBA OBSERVATIONS}

A $5 \mathrm{GHz}$ MERLIN observation of B2045+265 was made on 1997 November 30, with approximately $12.5 \mathrm{hr}$ total integration on source. The final map (Fig. 7) has an rms noise level of $0.146 \mathrm{mJy}^{-1}$ beam $^{-1}$ and an angular resolution of 60 mas. The data are well fitted by five point sources, with flux densities given in Table 3. However, there are some indications of additional emission around components $\mathrm{A}$ and $\mathrm{C}$ in the residual map, at the $2-3 \sigma$ level. The excess emission may indicate that the images are slightly resolved. If milliarcsecond-scale structure can be detected in the images, it can be used to put constraints on the lens model. The transformation matrix between corresponding positions in the resolved images provides crucial limits on the lensing potential by fixing its second derivatives at those points (see, e.g., Gorenstein et al. 1988). Observations with high angular resolution and dynamic range are needed to search for such structure. A $5 \mathrm{GHz}$ VLBA snapshot (35 minutes total integration on source) of this system was made on 1995 November 12. In this observation, which has an angular resolution of $\sim 1$ mas, the three brightest components are detected. Each component is unresolved, but 


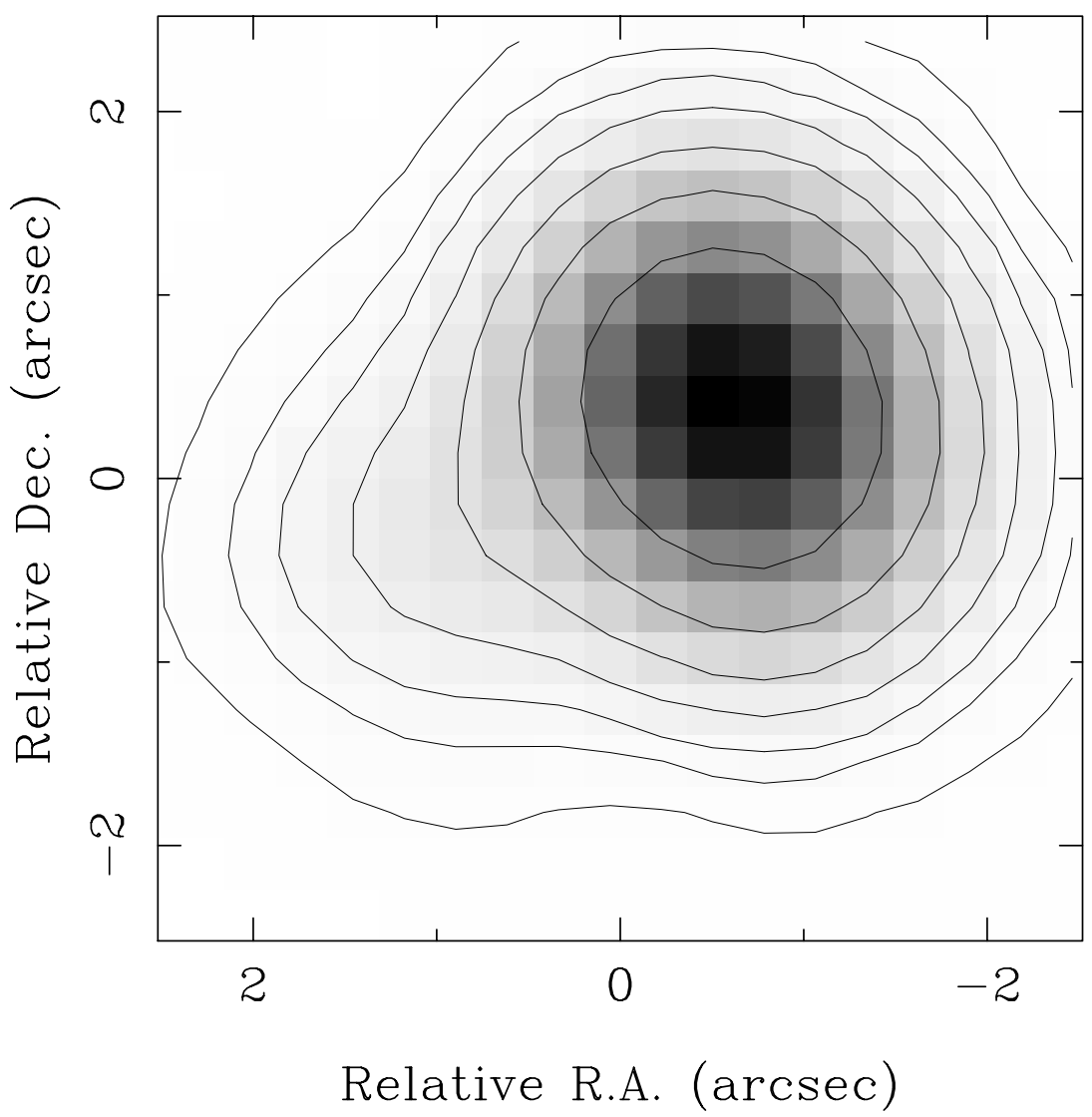

FIG. 4. - 1996 December $1.4 \mathrm{GHz}$ map. The contours are $(-2.5,2.5,5,10,20,40,80,160,320)$ times the rms noise level of $0.0784 \mathrm{mJy}^{\mathrm{beam}}{ }^{-1}$. Map made by fitting point-source components, with flux densities listed in Table 3 , to the $(u, v)$ data, and restoring with a 1 ". $5 \times 1$ 1". restoring beam.

the flux densities are only $\sim 60 \%$ of those seen in the VLA observations at the same frequency. Once again, this may indicate the presence of extended milliarcsecond-scale structure in this source that could be detected in more sensitive

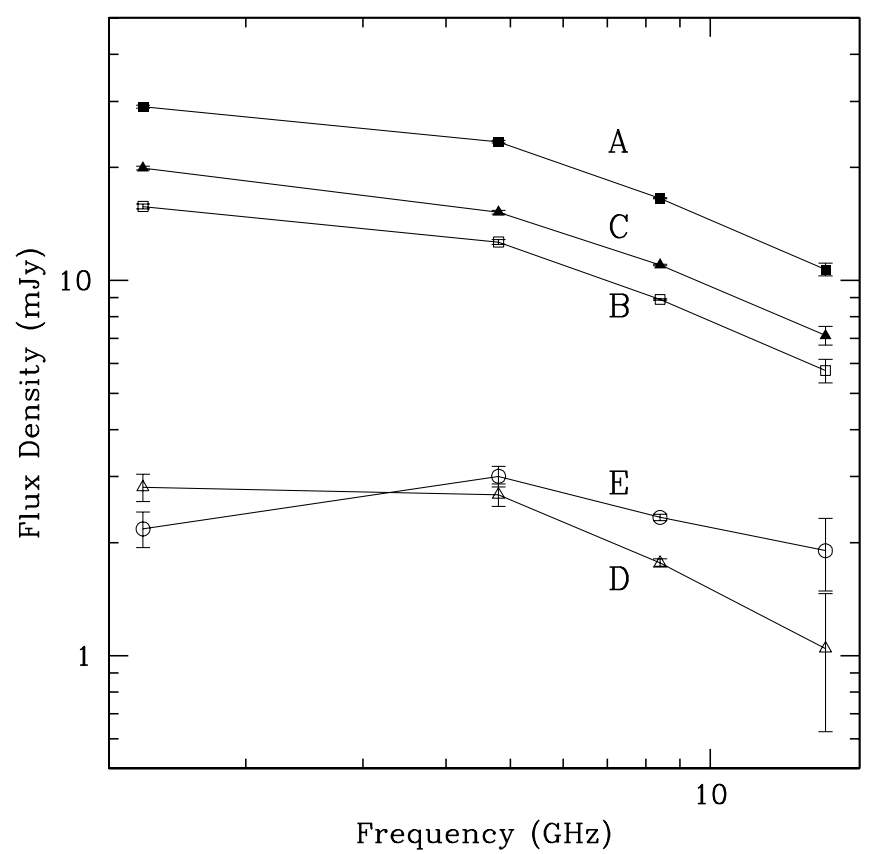

FIG. 5.-Radio spectra of the five components in the B2045+265 system. The error bars are $3 \sigma$ errors, based on the rms noise in the maps.

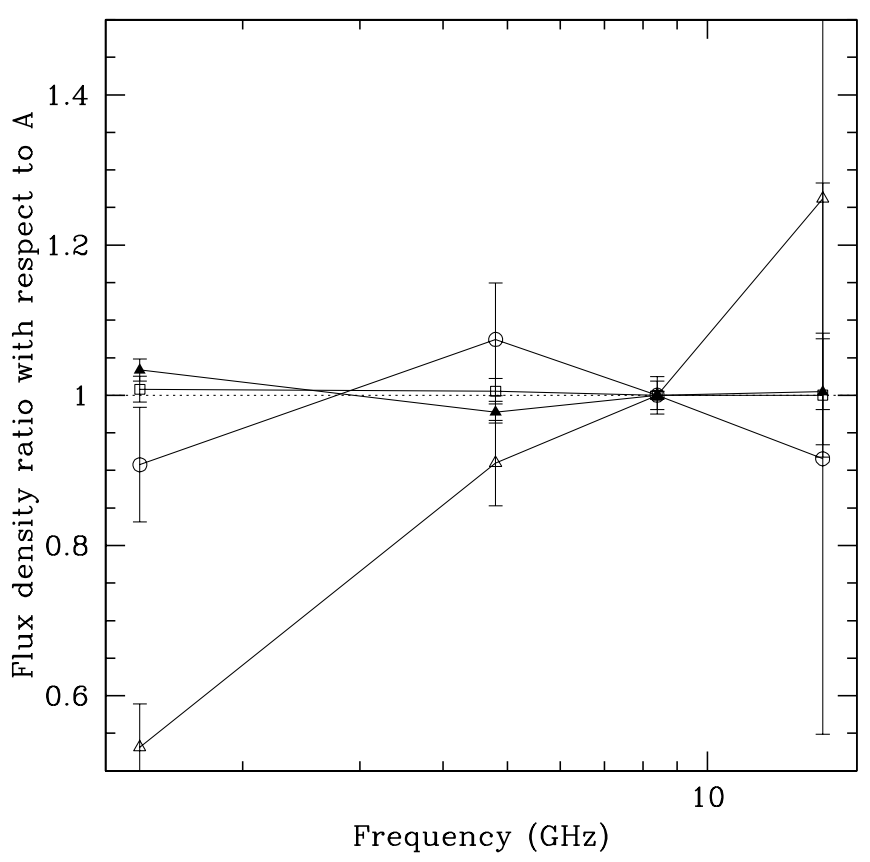

Fig. 6.-Flux density ratios for components B (open squares), C ( filled triangles), D (open circles), and $\mathrm{E}$ (open triangles) with respect to component A. All curves have been normalized to their $8.5 \mathrm{GHz}$ point. Error bars are calculated by propagating the rms noise in the maps at each frequency. 
TABLE 4

Component Flux Density Ratios

\begin{tabular}{ccccrr}
\hline \hline $\begin{array}{c}v \\
(\mathrm{GHz})\end{array}$ & \multicolumn{1}{c}{ Date } & \multicolumn{1}{c}{$\mathrm{B} / \mathrm{A}$} & $\mathrm{C} / \mathrm{A}$ & $\mathrm{D} / \mathrm{A}$ & \multicolumn{1}{c}{$\mathrm{E} / \mathrm{A}$} \\
\hline $1.4 \ldots \ldots$ & 1996 Dec 31 & $0.54 \pm 0.009$ & $0.69 \pm 0.010$ & $0.097 \pm 0.008$ & $0.075 \pm 0.008$ \\
$4.9 \ldots \ldots$. & 1996 Dec 31 & $0.54 \pm 0.009$ & $0.65 \pm 0.009$ & $0.11 \pm 0.008$ & $0.13 \pm 0.008$ \\
$8.5 \ldots \ldots$. & 1995 Sep 2 & $0.51 \pm 0.051$ & $0.80 \pm 0.059$ & $0.13 \pm 0.046$ & $0.099 \pm 0.046$ \\
& 1996 Dec 31 & $0.54 \pm 0.003$ & $0.66 \pm 0.003$ & $0.11 \pm 0.003$ & $0.14 \pm 0.003$ \\
$14.9 \ldots \ldots$ & 1995 Sep 11 & $0.59 \pm 0.072$ & $0.76 \pm 0.078$ & $0.079 \pm 0.062$ & $0.15 \pm 0.062$ \\
& 1996 Dec 31 & $0.54 \pm 0.044$ & $0.67 \pm 0.047$ & $0.098 \pm 0.046$ & $0.18 \pm 0.063$ \\
\hline
\end{tabular}

observations. Deep VLBA observations of B2045+265 have now been scheduled that may produce maps with sufficient dynamic range to detect extended structure in the images.

\section{INFRARED IMAGING}

\subsection{NIRC}

The system was imaged on 1996 July 31 using the Near Infrared Camera (NIRC; Matthews \& Soifer 1994) on the W. M. Keck I Telescope. Images were taken in both the $J$ and $K$ bands, with 45 exposures of 1 minute each in $K$ and 27 exposures of 1 minute each in $J$. In order to estimate the point-spread function (PSF), 18 exposures of a star at a distance of 1.47 were interleaved with the exposures on the lens in each band. The seeing was 0".45-0".90 in $K$ and 0 ".75-1".15 in $J$ during the observations.

The dark current level was subtracted from each image, and then sky subtraction and gain correction were performed. The sky and gain frames used for each image were constructed from images observed directly before and after it. The individual frames were aligned by centroiding on a star that appeared in each frame. For the highest sensitivity in the final images, all 45 frames in $K$ and all 27 frames in $J$ were combined into mosaics. The $K$-band mosaic is shown in Figure 8, and close-ups of the lens system in the two bands are shown in Figures 9 and 10. The two main features in the images of the lens system are a short arc, correspond-

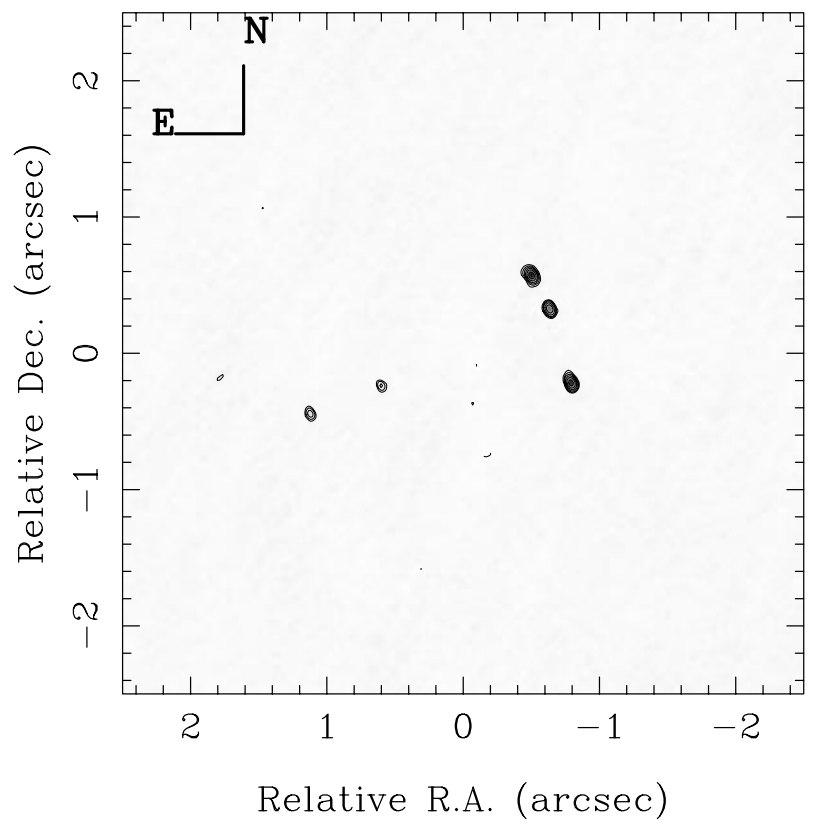

FIG. 7.-MERLIN map. Components A, B, and C may be slightly resolved. ing to the three brightest radio images, and the lensing galaxy, which is located 1"2 from the arc. Spectroscopy has shown that the object seen 2 " to the left of the lens system in Figure 8 is a star.

\subsection{NICMOS}

B2045+265 was observed with the Hubble Space Telescope using the Near Infrared Camera and MultiObject Spectrometer (NICMOS) on 1997 July 14. The NIC1 camera was used, which has a pixel scale of 43 mas. Two exposures were taken, giving a total exposure time of 2624 s. The images were subjected to the standard NICMOS calibration pipeline, involving bias and dark current subtraction, linearity and flat-field correction, photometric calibration, and cosmic-ray identification and removal. The final image (Fig. 11) clearly shows the three brightest lensed images and the lensing galaxy. The relative positions of components $\mathrm{A}, \mathrm{B}$, and $\mathrm{C}$ match those seen at radio wavelengths to within $00^{\prime \prime} 01$. In addition, the galaxy location matches the position of radio component $\mathrm{E}$ to within the errors. There appears to be low surface brightness emission between images $\mathrm{B}$ and $\mathrm{C}$, indicating the possibility that extended optical emission from the background source is being lensed into an arclike structure. The expected position of component $\mathrm{D}$ is marked in the figure.

\subsection{Photometry}

Because the NICMOS image has high spatial resolution, it is possible to compute magnitudes for the individual images of the background source in the F160W bandpass (roughly corresponding to the ground-based $H$ band). Magnitudes were calculated using both the DAOPHOT package (Stetson 1987) in IRAF $^{5}$ and the SExtractor package (Bertin \& Arnouts 1996). First, the magnitudes of the three bright lensed images were calculated using 0 ".26 diameter apertures. Next, an empirical PSF, constructed from radial profiles of stars in the field, was fitted to the emission from the lensed images and the star near the lensing galaxy. The scaled PSFs were then subtracted from the data, and the lens magnitude was calculated using a 1".9 diameter aperture. This aperture was chosen to match the largest image separation in the system, which is approximately equal to twice the Einstein radius of the lens. The aperture diameter corresponds to $7.9 h^{-1} \mathrm{kpc}$ at the redshift of the lens. The PHOTFNU header card was used to convert count rates in the image into flux densities into janskys, and then a Vega zero point of $1087 \mathrm{Jy}$ was assumed

\footnotetext{
${ }^{5}$ IRAF (Image Reduction and Analysis Facility) is distributed by the National Optical Astronomy Observatories, which are operated by the Association of Universities for Research in Astronomy under cooperative agreement with the National Science Foundation.
} 


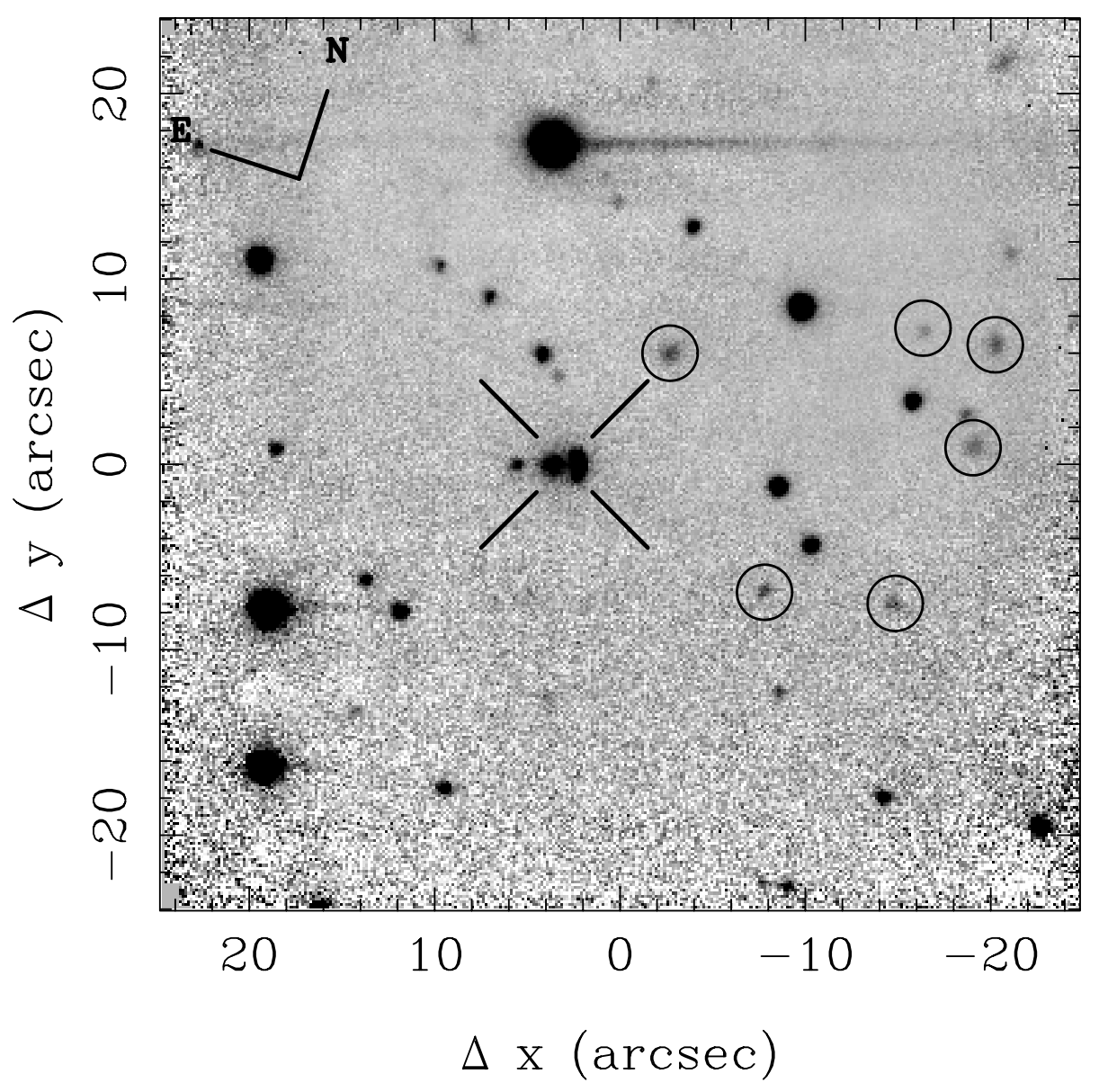

FIG. 8. - NIRC $K$-band mosaic of the B2045 +265 field. The lens system is marked with the crosshairs, with the lensing galaxy to the left and an arc of emission from the background source to the right. The object 2 " to the left of the galaxy is a star. The circled objects are extended and may be galaxies in a group at the redshift of the lens (see $\S 7.3$ ).

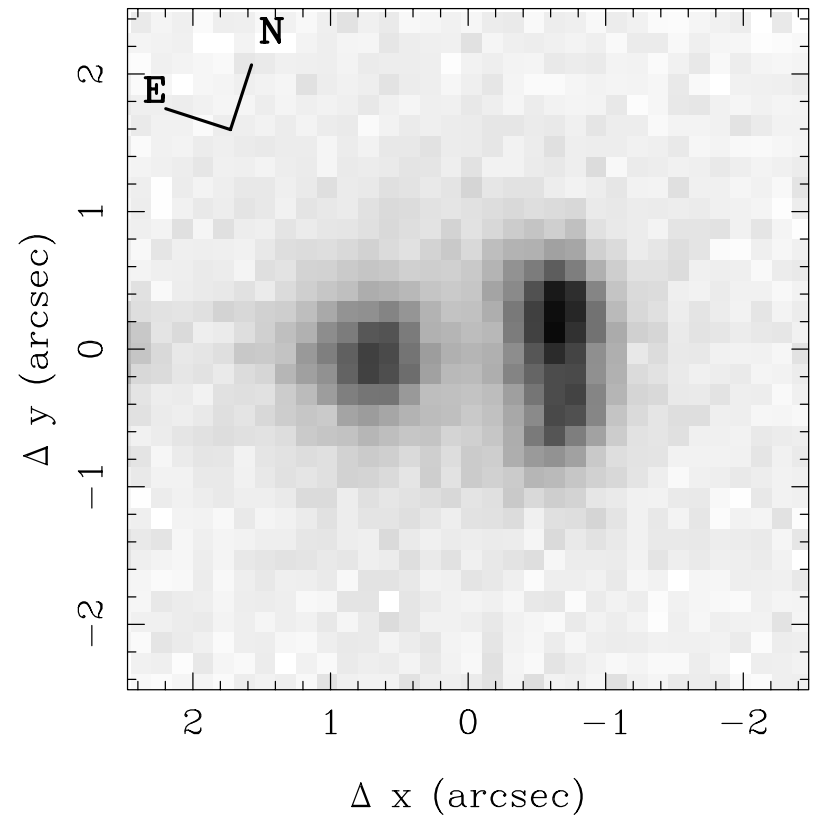

FIG. 9.-NIRC $K$-band image of B2045 +265 . The lensing galaxy is to the left, perhaps with some emission being contributed from component $\mathrm{D}$. An arc consisting of emission from components $\mathrm{A}, \mathrm{B}$, and $\mathrm{C}$ is to the right.

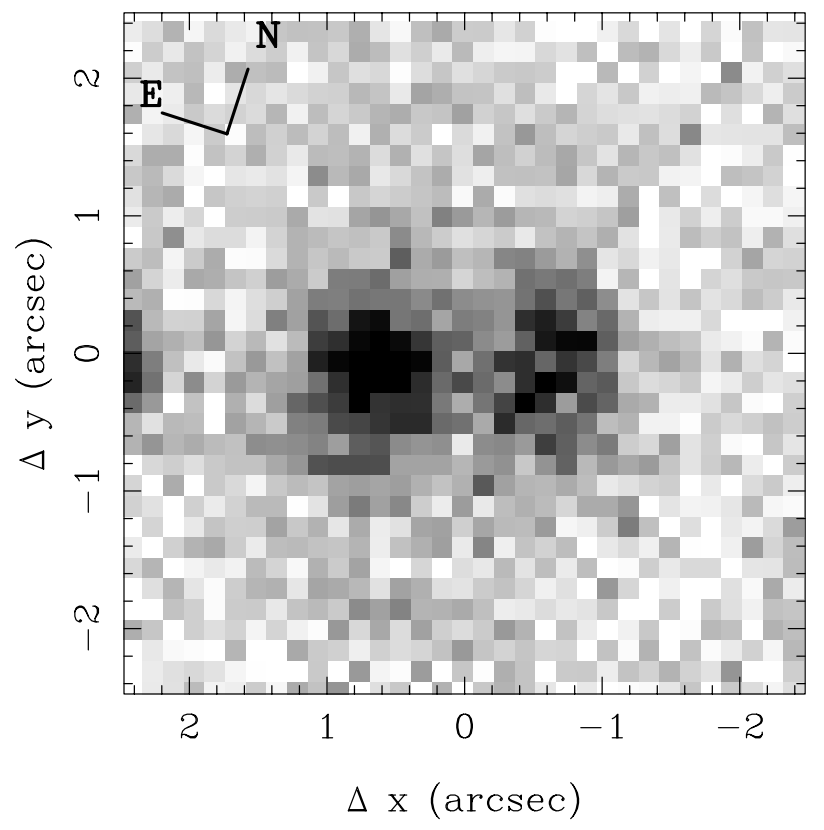

FIG. 10. - NIRC $J$-band image of B2045 + 265 


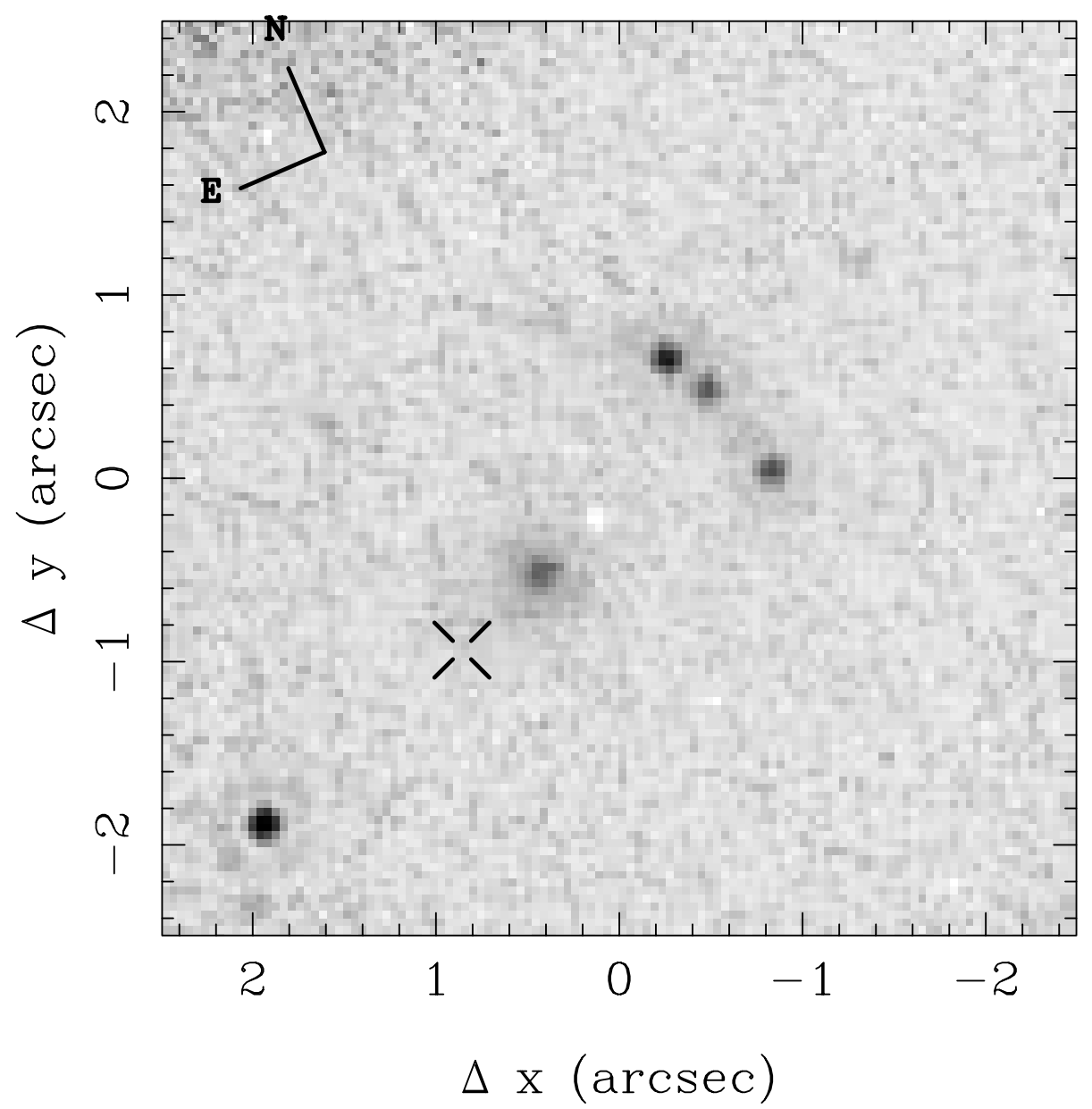

FIG. 11.-NICMOS F160W image of B2045 +265 . The extended object near the center of the image is the lensing galaxy. The crosshairs mark the expected position of component $\mathrm{D}$.

to get the F160W magnitudes. For comparison with the NIRC photometry (see below), the magnitude of the "arc" defined by the three lensed images of the background source was also computed. The DAOPHOT and SExtractor packages produced magnitudes that were consistent within the errors of the sky determination. The final magnitudes are given in Table 5. It should be possible to search for extinction caused by the lensing galaxy by comparing the F160W and radio flux density ratios of the lensed images. However, the uncertainties in the NICMOS component magnitudes are large $(\sim 0.2 \mathrm{mag})$, and thus the F160W flux density

TABLE 5

COMPONENT DATA (INFRARED OBSERVATIONS)

\begin{tabular}{cccccc}
\hline \hline Component & \multicolumn{1}{c}{$\Delta \alpha^{\mathrm{a}}$} & \multicolumn{1}{c}{$\Delta \delta^{\mathrm{a}}$} & \multicolumn{1}{c}{$J$} & $m_{\mathrm{F} 160 \mathrm{w}}{ }^{\mathrm{b}}$ & $K$ \\
\hline $\mathrm{A}^{\mathrm{c}} \ldots \ldots \ldots \ldots \ldots \ldots$ & 0.00 & 0.00 & $\ldots$ & 20.6 & $\ldots$ \\
$\mathrm{B}^{\mathrm{c}} \ldots \ldots \ldots \ldots \ldots \ldots$ & -0.13 & -0.23 & $\ldots$ & 21.0 & $\ldots$ \\
$\mathrm{C}^{\mathrm{c}} \ldots \ldots \ldots \ldots \ldots \ldots$ & -0.28 & -0.78 & $\ldots$ & 20.9 & $\ldots$ \\
Lens galaxy $^{\mathrm{d}} \ldots \ldots$. & +1.10 & -0.80 & 19.2 & 18.6 & 17.6 \\
"Arc $\ldots \ldots \ldots \ldots \ldots$ & $\cdots$ & $\ldots$ & 19.6 & 18.7 & 17.3 \\
\hline
\end{tabular}

${ }^{a}$ Relative positions in arcseconds calculated with respect to component $\mathrm{A}$ after $\mathrm{a}-23^{\circ}$ rotation was performed to match the radio image orientation.

${ }^{b}$ F160W magnitudes computed assuming that the Vega zero point is $1087 \mathrm{Jy}$.

c Magnitudes computed in a 0 ".26 diameter aperture.

d Magnitudes computed in a 1".9 diameter aperture. ratios, $\mathrm{B} / \mathrm{A}=0.69$ and $\mathrm{C} / \mathrm{A}=0.76$, are consistent with the radio flux density ratios at the $1 \sigma$ level. The upper limit on the brightness of a point source at the location of component $\mathrm{D}$ is $m_{\mathrm{F} 160 \mathrm{w}}>22 \mathrm{mag}$. This is not surprising, since component $\mathrm{D}$ should be 2.5 mag fainter than $\mathrm{A}$.

It is considerably more difficult to do photometry on the NIRC images, because the seeing disk is large compared with the component separations. Instead of finding magnitudes of the individual lensed images, the magnitude of the "arc" was calculated by centering a rectangular aperture of width 0.9 and height 1".8 on the "arc." The sky was estimated from regions directly to the north, west, and south of the aperture. Once again, the DAOPHOT package was used to subtract the emission from the lensed images and the star from the data before the lens galaxy magnitude was calculated. The photometric zero points were estimated by observing the infrared standard star SJ $9184(K=11.82$, $J=12.18 \mathrm{mag}$; S. E. Persson, private communication), which was observed in each band prior to the lens and PSF star exposures. The zero points in the two observed bands were $K_{0}=22.44$ and $J_{0}=22.92 \mathrm{mag}$. The $J$ and $K$ magnitudes of the lensing galaxy and "arc" are given in Table 5.

\section{OPTICAL SPECTROSCOPY}

Spectra of B2045+265 were taken with the Low Resolution Imaging Spectrograph (LRIS; Oke et al. 1995) on the Keck I Telescope on 1996 June 18-19. A 1" long slit 


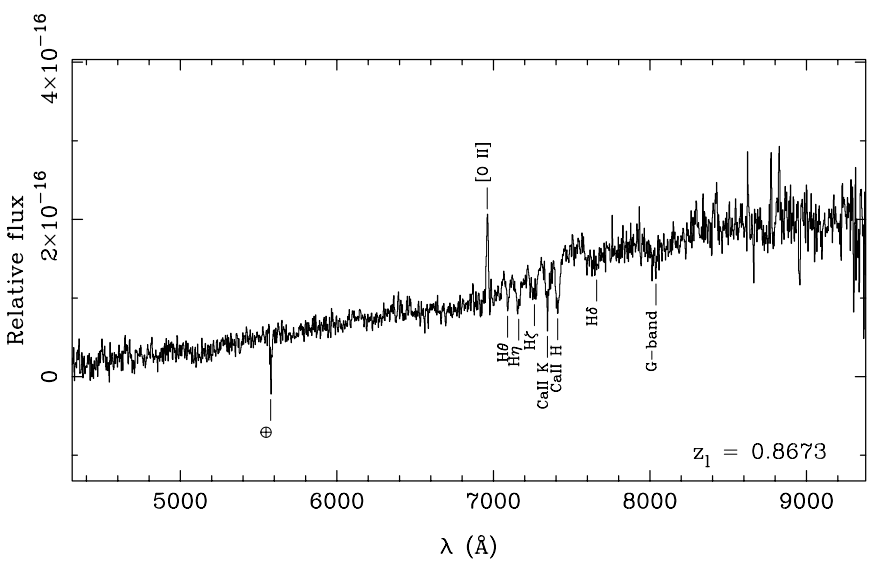

FIG. 12.-LRIS spectrum of the lensing galaxy from data taken on 1996 June 18-19.

and the 300 groove $\mathrm{mm}^{-1}$ grating were used, giving a pixel scale of $2.44 \AA$ pixel $^{-1}$. The slit was positioned at P.A. $=112^{\circ}$, in order to have the highest probability of spatially separating the emission of the background source from that of the lensing galaxy. Seven $1500 \mathrm{~s}$ exposures were taken: four on the first night, and three on the second night.

The spectra were reduced using standard IRAF tasks. The average seeing over the two nights was sufficiently

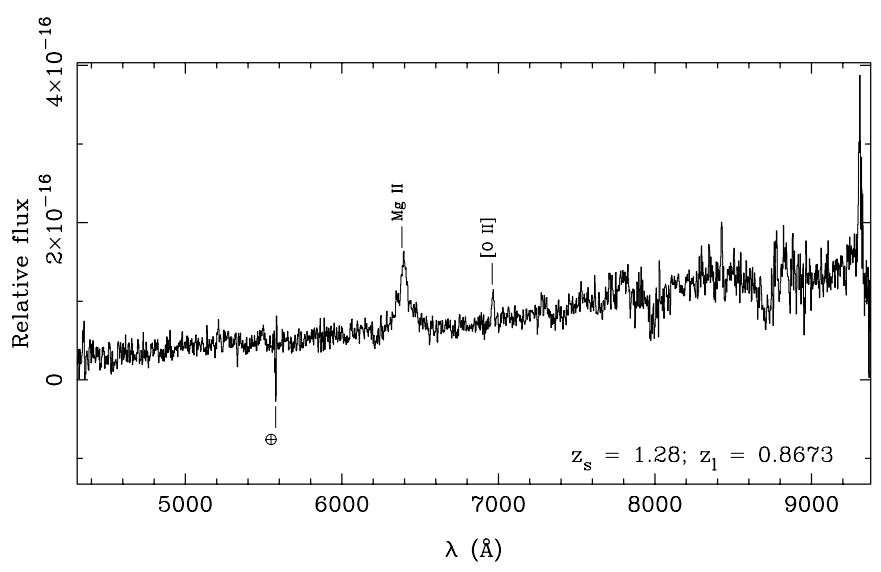

FIG. 13.- Spectrum of background source taken on 1996 June 18-19. There is some contamination from light from the lensing galaxy, which produces the $[\mathrm{O} \mathrm{II}]$ emission line.

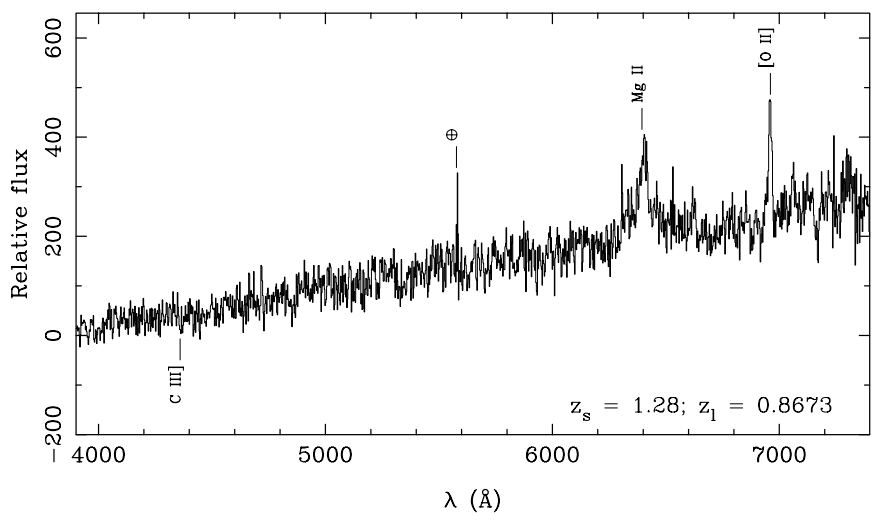

Fig. 14.-Wavelength-calibrated spectrum taken on 1996 September 15. Both source and lens features are seen in the spectrum. The position of the expected $\mathrm{C}$ III] emission is marked. The signal drops to zero blueward of $4000 \AA$. good to enable the extraction of separate spectra at the positions of the lensing galaxy and the brightest source emission. The spatial separation between lens and source spectra is 1 ..3, matching the separation between the lens and the three bright lensed images seen in the NICMOS image. The final spectra have a wavelength range 4309-9361 $\AA$. Flux calibration was performed using the standard star Feige 110 (Oke 1990), which was observed at the end of each night. An atmospheric absorption template was created by fitting a power law to the flux-calibrated spectrum of the BL Lac object 2155-304. This template was used to remove atmospheric features from the B2045+265 spectra. The individual exposures were weighted by the squares of their signal-to-noise ratios and combined to create the final lens and source spectra shown in Figures 12 and 13.

The lens system was reobserved with LRIS on 1996 September 15 with a setup similar to that used in the earlier observations, but with wavelength coverage 2344-7398. . Two $2000 \mathrm{~s}$ exposures were taken, and the data were reduced using a method similar to that described above. In this case, however, the chip response was not removed from the final spectrum. The seeing was worse than in the earlier observations; as a result, the source and lens spectra could not be extracted separately, and both source and lens features are seen in the final spectrum (Fig. 14).

The reduced spectrum of the lensing object is typical of an Sa galaxy (see, e.g., Kennicutt 1992). It shows [O II] $\lambda 3727$ emission at 6962 , Ca II K $\lambda 3934$ absorption at 7345 , Ca II H $\lambda 3968$ absorption (possibly blended with $\mathrm{H} \epsilon$ 23970) at $7411 \AA$, and $\mathrm{H} \eta$ and $\mathrm{H} \theta \lambda \lambda 3835,3797$ absorption at 7159 and $7092 \AA$, respectively. These spectral features establish the lens redshift as $z_{l}=0.8673 \pm 0.0005$, where the uncertainty in the redshift is determined from the rms scatter in the redshifts derived from the [O II], $\mathrm{Ca}$ II $\mathrm{K}, \mathrm{H} \eta$, and $\mathrm{H} \theta$ lines. The source spectrum shows a broad emission line at $\lambda=6396 \AA$. No other broad emission features are seen in the spectrum, which ranges from 4300 to nearly $9400 \AA$. We identify the line as $\mathrm{Mg}$ II $\lambda 2800$, which implies $z_{s}=1.28$, because it is the only broad emission line that is so isolated in typical quasar spectra (see, e.g., Boyle 1990; Francis et al. 1991). The second set of spectra were taken in order to search for C III] $\lambda 1909$ emission, expected to fall at $\sim 4350$ $\AA$. No line was seen at this position, although the sensitivity in this part of the spectrum is low, and the presence of a weak line is not ruled out. Other possible identifications for the observed emission line (e.g., $\mathrm{H} \beta, \mathrm{C}$ III], C IV, Ly $\alpha$ ) would imply the presence of strong emission lines in parts of the spectrum in which the signal-to-noise ratio is high, none of which we see. We conclude that the line is correctly identified and the source lies at redshift $z_{s}=1.28 \pm 0.01$, where the uncertainty in the redshift is estimated from uncertainties in finding the line centroid in both the emitted and the observed frames.

\section{SOURCE VARIABILITY}

In order for a lens system to be used to measure $H_{0}$, the background source must be variable, so that time delays can be measured. We use two methods to search for evidence of variability in the background source in B2045 +265. The first is to examine the component flux density ratios at different epochs. The ratios are not affected by errors in the absolute flux calibration, and so any detected changes in the flux density ratios reflect actual 
changes in the flux density of the background source. A comparison of the $8.5 \mathrm{GHz}$ observations made in $1995 \mathrm{Sep}-$ tember with those made in 1996 December shows small changes in the ratios of the component flux densities, but not at a significant level.

The second method is to monitor the total $22 \mathrm{GHz}$ flux density of the B2045 + 265 lens system. Daily observations of the system were made with the $40 \mathrm{~m}$ telescope of the Owens Valley Radio Observatory during the periods 1996 May 18-September 3 and 1996 November 4-22. The data were calibrated using the CMBPROG package (Leitch 1998). A $15 \% \pm 3 \%$ change in total flux density was observed in 1996 November over a period of less than a week. Because several other sources being monitored showed no change in flux density during the same period, the observed change in flux density may indicate variability in the background source. However, it is also possible that the detected variability is due to changes in component $\mathrm{E}$, which has a radio spectrum that is flatter than that of the lensed images. To determine which of the components is varying requires high angular resolution monitoring of the system with the VLA.

\section{DISCUSSION}

\subsection{The Nature of Component $E$}

The detection of five unresolved radio components in the B2045 + 265 system raises the possibility that component $\mathrm{E}$ is the fifth lensed image of the background source. We believe, however, that component $\mathrm{E}$ is instead associated with the lensing galaxy. Although standard lensing models can produce five images in configurations similar to that seen in the B2045+265 system (see, e.g., Blandford \& Narayan 1992), the central fifth image tends to be highly demagnified with respect to the other images. This behavior is not seen in B2045 + 265; in fact, component $\mathrm{E}$ is brighter than component $\mathrm{D}$ at high frequencies. In addition, the radio spectrum of component $\mathrm{E}$ (Figs. 5 and 6) differs sufficiently from the spectra of the other four components to suggest that component $E$ is not related to the other components. Finally, the radio emission of component $\mathrm{E}$ is spatially coincident with the infrared emission from the lensing galaxy (see $\S 4.2$ ). We therefore conclude that component $\mathrm{E}$ is indeed associated with the lensing galaxy. It thus appears that we have discovered a radio galaxy lensing a radio-loud quasar. Of previously known lens systems, only $2016+112$ (Lawrence et al. 1984) may have radio emission associated with a lensing galaxy. However, recent observations of $2016+112$ suggest that the radio emission of its component C1 may be lensed emission from a (separate) background source (Garrett et al. 1996).

The flat radio spectrum of component $\mathrm{E}$ in $\mathrm{B} 2045+265$ is typical of an active galactic nucleus. If component $\mathrm{E}$ is, indeed, associated with the lensing galaxy, then the radio position of $E$ gives an accurate location for the nucleus of the lensing galaxy. The location of the center of the lensing galaxy relative to the lensed images is an important component in models of the lensing potential (see § 7.2). An a priori knowledge of the relative position of the center of the lensing galaxy provides strong constraints on lens models.

\subsection{Preliminary Lens Model}

We model the lens potential as a singular isothermal sphere potential with "mixed" shear (Kochanek 1991), in which the scaled lensing potential can be expressed as

$$
\psi=b r+\gamma b r \cos 2\left(\theta-\theta_{\gamma}\right) .
$$

Locations in the image plane relative to the lens center are given by $\boldsymbol{x}=(r, \theta)=(x, y)$. The critical radius of the isothermal sphere is given by $b$, and $\gamma$ and $\theta_{\gamma}$ give the magnitude of the shear and its position angle. In the modeling process we have used an astronomical rather than mathematical coordinate system, such that $x \equiv \Delta \alpha$ and $y \equiv \Delta \delta$. The polar coordinates are defined in the usual way, with $r=\left(x^{2}+y^{2}\right)^{1 / 2}$ and $\theta=\arctan (y / x)$. This results in a lefthanded coordinate system.

Although the image positions are defined relative to the position of component A, they are treated as absolute positions. Thus, both the lens position $\left(x_{l}, y_{l}\right)$ and source position $\left(x_{s}, y_{s}\right)$ are included as parameters in the model. We assume that component $\mathrm{E}$ marks the nucleus of the lensing galaxy and fix the lens position at that location, leaving five varying parameters and eight observational constraints ( $x$ and $y$ for each of the four images).

A downhill simplex routine (Press et al. 1992) is used to minimize the difference between the observed and model positions, expressed as

$$
\chi^{2}=\sum_{i}\left(\frac{\left|x_{m}-x_{o}\right|^{2}}{\sigma_{x}^{2}}\right),
$$

where the subscripts $m$ and $o$ refer to the model and observed values, respectively. The positional errors are defined to be the $8.5 \mathrm{GHz}$ beam size divided by the signalto-noise ratio of the component in the $8.5 \mathrm{GHz}$ map. To avoid biases introduced by the choice of starting values, we repeat the process with a grid of choices of initial conditions containing $3^{n}$ values, where $n$ is the number of varying parameters in the model fitting. The best-fit parameters for the model are given in Table 6, and the model is shown graphically in Figure 15. The observed image positions are recovered by this simple model of the lensing potential, with rms image displacements of less than 20 mas.

Radio images are unaffected by microlensing, so it is possible to use the flux density ratios of the images to constrain the model of the lensing potential further. The observed flux density ratios, however, are difficult to reproduce with a simple model such as the one presented above. Although this simple model is adequate to recover the four image positions, it does not reproduce the relative magnifications of images A, B, and C. The reason for this is easy to see. If,

TABLE 6

\begin{tabular}{|c|c|}
\hline Parameter & Value \\
\hline$b$ & 1.076 \\
\hline$\gamma \ldots$ & 0.104 \\
\hline & 69.4 \\
\hline$x_{l}^{\mathrm{a}} \ldots \ldots$ & $+1 " 12^{\mathrm{b}}$ \\
\hline$y_{l}^{\mathrm{a}} \ldots \ldots$ & $-0.82^{b}$ \\
\hline$x_{s}{ }^{\mathrm{a}} \cdots \cdots$ & +0.72 \\
\hline 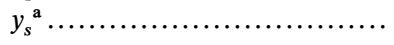 & -0.67 \\
\hline
\end{tabular}

LENS MODEL

${ }^{\text {a }}$ The positions relative to component $\mathrm{A}(x$, $y)$ and $\theta_{\gamma}$ are defined in a left-handed coordinate system. See $\S 7.2$.

${ }^{b}$ Held fixed during model fitting. 


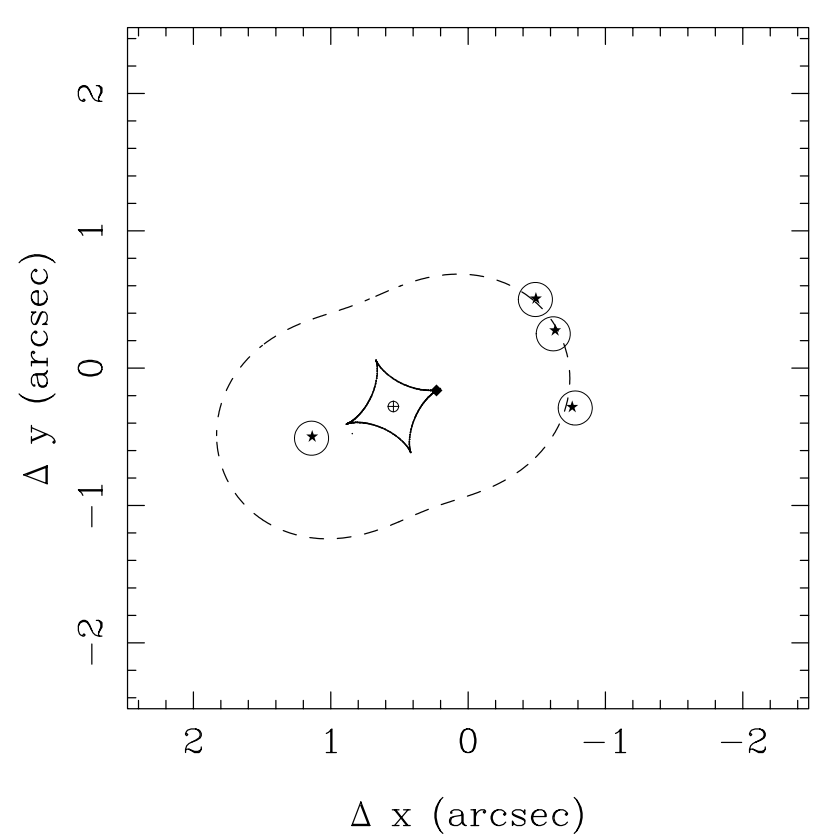

FIG. 15.- - Lens model showing critical curve (dashed line), caustic curve (solid line), lens center (circled plus sign), source position (filled diamond), and model image positions (stars). The four circles are centered on the observed image positions.

as we have assumed, the potential is smooth, then we can perform a Taylor expansion of it about the location of image B. If we approximate the images as collinear, then the highest order essential terms in this expansion are quadratic in the coordinate perpendicular to the A-C line and quartic parallel to this line. Higher order terms are, by assumption, ignorable. It is then possible to show that in the limit of small image separations there are several scaling laws, including $S_{B} \sim S_{A}+S_{C}$, i.e., the flux of the central image approaches the sum of the fluxes of the other two images (see, e.g., Kovner \& Paczyński 1988; Blandford 1990). This is clearly violated in this source, which indicates strongly that the potential is not smooth on the scale of the A-C image separation.

In order to improve on our model, we have introduced higher order terms in the potential such as might be produced by a local mass perturbation. Specifically, we expand the potential around images $\mathrm{A}$ and $\mathrm{C}$ to third order along the A-C line and to fourth order around image B. We then impose continuity of the potential and its first two derivatives midway between $A$ and $B$ and midway between $B$ and C. There are then three observable quantities, the ratio of the image separations and the ratios of the image fluxes, that can be used to solve for the coefficients in these Taylor expansions. Performing this exercise leads to the conclusion that adding a small, positive mass perturbation centered on a point located 0 ."11 from B toward C suffices to recover the flux ratios as well as the image locations. (This perturbation might be caused by a spiral arm, for example; see Mao \& Schneider 1997.) The ratio of the (almost certainly unmeasurable) time delays $\Delta t_{\mathrm{BA}} / \Delta t_{\mathrm{BC}}$ is now found to be 0.2 ; however, the delay $\Delta t_{\mathrm{BD}}$ should be quite robust to this perturbation within a given global model. In conclusion, none of the existing observations challenge the gravitational lens interpretation that we present here.

\subsection{Properties of the Lensing Galaxy}

Properties of the lensing galaxy can be derived by using the fact that it is acting as a gravitational lens. For example, the mass within the Einstein ring of a lens is

$$
M_{\mathrm{E}} \approx 1.24 \times 10^{11}\left(\frac{\theta_{\mathrm{E}}}{1^{\prime \prime}}\right)^{2}\left(\frac{D}{1 \mathrm{Gpc}}\right) M_{\odot}, \quad D \equiv \frac{D_{l} D_{s}}{D_{1 \mathrm{~s}}}
$$

(see, e.g., Blandford \& Narayan 1992), where $\theta_{\mathrm{E}}$ is the angular radius of the Einstein ring and $D_{l}, D_{s}$, and $D_{1 \mathrm{~s}}$ are the angular diameter distances between observer and lens, observer and source, and lens and source, respectively. The angular diameter distance between two objects at $z_{i}$ and $z_{j}$ $\left(z_{i}<z_{j}\right)$ is given by

$$
D_{i j}=\frac{c}{H_{0}\left(1+z_{j}\right)\left|\Omega_{k}\right|^{1 / 2}} \sin n\left[\left|\Omega_{k}\right|^{1 / 2} \mathscr{I}\left(z_{i}, z_{j}\right)\right]
$$

(Carroll, Press, \& Turner 1992), where

$$
\mathscr{I}\left(z_{i}, z_{j}\right)=\int_{z_{i}}^{z_{j}} \frac{d z}{\left[(1+z)^{2}\left(1+\Omega_{M} z\right)-z(2+z) \Omega_{\Lambda}\right]^{-1 / 2}}
$$

and $\sin n x$ is defined as $\sinh x$ for $\Omega_{k}<0, x$ for $\Omega_{k}=0$, or $\sin x$ for $\Omega_{k}>0$, respectively. The $\Omega$ terms are defined in the standard fashion:

$$
\Omega_{M}=\frac{8 \pi G}{3 H_{0}^{2}} \rho_{0}, \quad \Omega_{\Lambda}=\frac{\Lambda}{3 H_{0}^{2}}, \quad \Omega_{k}=-\frac{k c^{2}}{R_{0}^{2} H_{0}^{2}} .
$$

The integral $\mathscr{I}$ has an analytic solution for $\Omega_{\Lambda}=0$, and if $\Omega_{M}=1$, the angular diameter distance acquires the simple form

$$
D_{i j}=\frac{2 c}{H_{0}} \frac{1}{\left(1+z_{j}\right)}\left[\frac{1}{\left(1+z_{i}\right)^{1 / 2}}-\frac{1}{\left(1+z_{j}\right)^{1 / 2}}\right] .
$$

For B2045 +265, the lens redshift is secure. The source redshift, based on one emission line, is less certain. For this reason, we express the physical quantities derived in this section as functions of the source redshift. We will also assume, for ease of discussion, an Einstein-de Sitter cosmology. The effects of varying the source redshift and the cosmological model are presented in Table 7. The angular diameter distances for this system are $D_{l}=860 h^{-1} \mathrm{Mpc}$ and

$$
\frac{D_{s}}{D_{1 \mathrm{~s}}}=\frac{1.8673\left[\left(1+z_{s}\right)^{1 / 2}-1\right]}{\left(1+z_{s}\right)^{1 / 2}-1.8673} .
$$

The mass enclosed within the Einstein ring radius is calculated by assuming that the maximum image separation is a measure of $2 \theta_{\mathrm{E}}$, i.e., that $\theta_{\mathrm{E}}=0.95$. This assumption yields $M_{\mathrm{E}} \approx 9.6 \times 10^{10}\left(D_{s} / D_{\mathrm{ls}}\right) h^{-1} \quad M_{\odot}$ for the $\mathrm{B} 2045+265$ system. Note that $M_{\mathrm{E}}$ represents the total mass contained in the cylinder with projected radius $R_{\mathrm{E}}$. The speed of a particle moving in a circular orbit $\left(v_{\text {circ }}\right)$ at the Einstein ring radius is derived from $M_{\mathrm{E}}$ by assuming that the mass distribution of the lensing galaxy is an isothermal sphere. A simple calculation is used to convert $M_{\mathrm{E}}$ to the mass inside the sphere of radius $R_{\mathrm{E}}$. The value for $v_{\text {circ }}$ follows directly from the mass inside the sphere. The luminosity of the lens inside $R_{\mathrm{E}}$ can be estimated by converting its $K$ magnitude to rest-frame $M_{B}$ and $M_{V}$ magnitudes. The $k$-corrections for the lens redshift and rest-frame $V-K$ and $B-V$ colors for typical Sa galaxies are taken from Poggianti (1997). The 
TABLE 7

LENS PARAMETERS

\begin{tabular}{|c|c|c|c|c|c|c|c|c|c|}
\hline $\begin{array}{c}\text { Line } \\
\text { Identification }\end{array}$ & $z_{s}$ & $\Omega_{M}$ & $\Omega_{\Lambda}$ & $\begin{array}{c}D_{l} \\
\left(h^{-1} \mathrm{Mpc}\right)\end{array}$ & $\begin{array}{c}D_{s} \\
\left(h^{-1} \mathrm{Mpc}\right)\end{array}$ & $\begin{array}{c}D_{1 \mathrm{~s}} \\
\left(h^{-1} \mathrm{Mpc}\right)\end{array}$ & $\begin{array}{c}M_{\mathrm{E}} \\
\left(10^{11} h^{-1} M_{\odot}\right)\end{array}$ & $\begin{array}{c}\left(M / L_{B}\right)_{\mathrm{E}} \\
{\left[h\left(M / L_{B}\right)_{\odot}\right]}\end{array}$ & $\begin{array}{c}v_{\text {circ }} \\
\left(\mathrm{km} \mathrm{s}^{-1}\right)\end{array}$ \\
\hline \multirow{3}{*}{ Mg пा......... } & 1.28 & 1.0 & 0.0 & 860 & 890 & 180 & 4.7 & 20 & 570 \\
\hline & & 0.2 & 0.0 & 1000 & 1100 & 240 & 5.3 & 16 & 560 \\
\hline & & 0.2 & 0.8 & 1200 & 1300 & 340 & 5.1 & 11 & 500 \\
\hline \multirow[t]{3}{*}{$\mathrm{C}$ III] $\ldots \ldots \ldots$} & 2.35 & 1.0 & 0.0 & 860 & 810 & 330 & 2.3 & 10 & 400 \\
\hline & & 0.2 & 0.0 & 1000 & 1200 & 470 & 2.8 & 9 & 400 \\
\hline & & 0.2 & 0.8 & 1200 & 1300 & 650 & 2.6 & 6 & 360 \\
\hline \multirow[t]{3}{*}{$\mathrm{C}$ IV .......... } & 3.13 & 1.0 & 0.0 & 860 & 740 & 350 & 2.0 & 8 & 370 \\
\hline & & 0.2 & 0.0 & 1000 & 1100 & 530 & 2.5 & 8 & 380 \\
\hline & & 0.2 & 0.8 & 1200 & 1200 & 700 & 2.3 & 5 & 340 \\
\hline \multirow[t]{3}{*}{$\operatorname{Ly} \alpha \ldots \ldots \ldots$} & 4.26 & 1.0 & 0.0 & 860 & 640 & 337 & 1.8 & 7 & 360 \\
\hline & & 0.2 & 0.0 & 1000 & 1100 & 540 & 2.3 & 7 & 370 \\
\hline & & 0.2 & 0.8 & 1200 & 1100 & 690 & 2.1 & 5 & 330 \\
\hline
\end{tabular}

resulting luminosities are $\left(L_{V}\right)_{\mathrm{E}}=2.92 \times 10^{10} \mathrm{~h}^{-2} L_{\odot, V}$ and $\left(L_{B}\right)_{\mathrm{E}}=2.36 \times 10^{10} h^{-2} L_{\odot, B}$. The rest-frame $B$ band mass-to-light ratio becomes $\left(M / L_{B}\right)_{\mathrm{E}}=4.1\left(D_{\mathrm{s}} / D_{\mathrm{ls}}\right) h$ $\left(M / L_{B}\right)_{\odot}$.

Inserting the source redshift derived from the optical spectra (§ 5) yields a lens mass of $M_{\mathrm{E}} \approx 4.7 \times 10^{11} h^{-1} M_{\odot}$ within the Einstein ring radius $\left(\sim 4 h^{-1} \mathrm{kpc}\right.$ at the redshift of the lens). This mass implies a circular velocity at $R_{\mathrm{E}}$ of $570 \mathrm{~km} \mathrm{~s}^{-1}, 2-3$ times the velocities seen in both nearby (see, e.g., Rubin et al. 1985) and more distant $(0.1<z<1$; Vogt et al. 1996) spiral galaxies. The Einstein ring mass-tolight ratios are $\left(M / L_{V}\right)_{\mathrm{E}}=16 h\left(M / L_{V}\right)_{\odot}$ and $\left(M / L_{B}\right)_{\mathrm{E}}=20$ $h\left(M / L_{B}\right)_{\odot}$. These mass-to-light ratios are not unusual for lens systems (see, e.g., Fig. 7 of Keeton, Kochanek, \& Falco 1998), although they are higher than expected given the redshift of the lens and assumptions about the luminosity evolution of lens galaxies (Keeton et al. 1998). The large lensing mass required to produce the observed image separation is a result of the relative closeness of the background source to the lens in redshift space. There are several possible explanations for the unusually high projected mass.

Compact groups of galaxies associated with the primary lensing galaxies have been discovered in recent observations of two lens systems $(1115+080$ and B1422 +231 ; Kundić et al. 1997a, 1997b; Tonry 1998). We are conducting observations of the B2045 + 265 field to search for evidence of a group associated with the lensing galaxy (see also Fig. 8). The high contamination rate by field galaxies $(\sim 85 \%$ at $z \sim 1$; Postman, Lubin, \& Oke 1998), however, makes highredshift groups difficult to detect. If such a group exists and is gravitationally bound, its gravitational potential can enhance the image splitting by the lensing galaxy. In such a scenario, we would overestimate the mass of the lensing galaxy.

Another explanation for the high projected mass inside the Einstein ring is that the lens may be one of a close pair of possibly merging galaxies. This situation has been observed in the case of the CLASS lens $1608+656$. The initial observations revealed an Einstein ring projected mass of $M_{\mathrm{E}} \approx 3.1 \times 10^{11} h^{-1} M_{\odot}$, corresponding to $v_{\text {circ }}=$ $440 \mathrm{~km} \mathrm{~s}^{-1}$ (Myers et al. 1995; Fassnacht et al. 1996). Later Hubble Space Telescope imaging showed that the lens is composed of a very close pair of galaxies, explaining the high mass and ellipticity required for the $1608+656$ system (Jackson, Nair, \& Browne 1997). We are unable to determine unequivocally whether two galaxies are present in the
B2045 + 265 system because the sensitivity of our NICMOS observation is too low. More sensitive NICMOS and Wide Field Planetary Camera 2 observations of B2045 + 265 are needed to test the two-galaxy hypothesis.

A third possibility is that the source redshift or the assumed cosmology (or both) is in error. We examined the results of varying the source redshift by assigning different identifications to the emission line seen in Figure 13. We then recalculated the physical parameters associated with the lens in three different cosmological models: $\left(\Omega_{M}, \Omega_{\Lambda}\right)=$ $(1,0),(0.2,0)$, and $(0.2,0.8)$. We find that the effects of changing $z_{s}$ are larger than those produced by changing the cosmological model. Of the alternative identifications of the emission line in the source spectrum, the choice of Ly $\alpha$ produces lens galaxy properties that are the closest to those associated with normal nearby spiral galaxies (Table 7). However, no sign of a $L y \alpha$ forest is seen on the shortwavelength side of the emission line, even after subtracting the estimated contribution of the lens galaxy from the source spectrum. The absence of other emission lines in the spectrum $(\S 5)$ and the lack of a Ly $\alpha$ forest argue against the identification of the emission line as Ly $\alpha$. More sensitive spectroscopy of this system must be conducted to determine the source redshift unambiguously.

\subsection{Prospects for Measuring $H_{0}$}

A lens system can be used to estimate $H_{0}$ by comparing the time delays predicted by the lens model with the observed time delays. The time delays are a function of the lensing potential, the source and image positions, the redshifts of the source and lens, and the world model (through the angular diameter distances):

$$
\Delta t_{i}=\left(1+z_{l}\right) \frac{D_{l} D_{s}}{c D_{\mathrm{ls}}}\left[\frac{1}{2}\left|\boldsymbol{x}_{i}-\beta\right|^{2}-\psi\left(\boldsymbol{x}_{i}\right)\right],
$$

where $\boldsymbol{x}_{i}=\left(x_{i}, y_{i}\right)$ is the angular position of the $i$ th lensed image and $\beta=\left(x_{s}, y_{s}\right)$ is the position of the source. The observable quantity is the difference in the time delays, $\Delta t_{i j} \equiv \Delta t_{i}-\Delta t_{j}$. The predicted delays are proportional to $h^{-1}$ through the ratio of angular diameter distances, so if it is possible to measure delays, the ratio of the predicted and observed delays will give $h$. For the model given in $\S 7.2$, the components are expected to vary in the order C, A, B, D. The predicted delays between components $\mathrm{A}, \mathrm{B}$, and $\mathrm{C}$ are small: $\Delta t_{\mathrm{AC}}=6.5 h^{-1} \mathrm{hr}$ and $\Delta t_{\mathrm{BC}}=7.7 h^{-1} \mathrm{hr}$. The 
delays between each of the three bright components and component D are all on the order of $142 h^{-1}$ days. It will be challenging to measure the short time delays because radioloud quasars typically do not vary significantly on those timescales. However, with high-sensitivity observations, it will be quite possible to measure the long delays in this system, if the background source is variable.

\section{SUMMARY AND FUTURE WORK}

We have discovered a new four-image gravitational lens in the second phase of CLASS. The B2045 + 265 lens system is unusual in several respects. First, it is, with the possible exception of the puzzling $2016+112$ system, the first known gravitational lens system in which both the background source and the lensing galaxy are radio sources. The radio emission from the lens, presumably from an active nucleus, can be used to locate the center of the lensing galaxy with high precision, which provides a strong constraint for lens models. Second, the flux density ratios of the three brightest lensed images are inconsistent with the predictions of simple models of lensing potentials. This result may indicate that there is significant structure in the mass distribution of the lens. Finally, the projected mass (and mass-to-light ratio) inside the Einstein ring of the lens is unusually high for an Sa galaxy. This may imply that there is a significant amount of dark matter associated with this galaxy, that the lens actually consists of a close pair of galaxies, or that the overall mass distribution of an associated group of galaxies is contributing to the lensing potential. Alternatively, the source redshift may be misidentified.

There are intriguing hints that the images of the background source may be resolved by high angular resolution observations. We have obtained high dynamic range VLBA observations that can be used to search for this possible extended milliarcsecond-scale structure. If such structure is detected, the transformation matrices between the lensed images of the background source provide strong constraints on the lensing model. These data may explain the unusual flux density ratios among the three bright images. The VLBA observations also will provide a more accurate position for the center of the lensing galaxy. In addition, more sensitive Hubble Space Telescope and spectroscopic observations can provide further details about the lensing galaxy and background source. The goal of these observations is to develop a well-constrained model of the lensing potential. A program of monitoring can be used to search for time delays in the system. Any measured delays can be combined with the lens models to yield a measurement of $H_{0}$.

We are indebted to the VLA analysts and operators, to Terry Stickel and Wayne Wack for heroic operation of the Keck Telescopes, and to the Keck Observatory and STScI staff. C. D. F. thanks Erik Leitch for generously spending many hours in instruction in the use of the OVRO $40 \mathrm{~m}$ telescope and for writing the CMBPROG software. The operation of the $40 \mathrm{~m}$ telescope would be impossible without the dedication and knowledge of Russ Keeney and Mark Hodges. We are grateful to Gerry Neugebauer, Lee Armus, and Aaron Evans for their expert assistance during the reduction of the NIRC and NICMOS data. We thank Lori Lubin, D. Wardell Hogg, Mike Pahre, Mark Metzger, and Chung-Pei Ma for useful discussions and comments on the manuscript. We thank the anonymous referee for helpful suggestions on how to improve the paper. This work is supported by the NSF under grant AST 94-20018 and by the European Commission, TMR Program, Research Network contract ERBFMRXCT96-0034 "CERES."

\section{REFERENCES}

Bertin, E., \& Arnouts, S. 1996, A\&A, 117, 393

Blandford, R. D. 1990, QJRAS, 31, 305

Blandford, R. D., \& Narayan, R. 1992, ARA\&A, 30, 311

Boyle, B. J. 1990, MNRAS, 243, 231

Carroll, S. M., Press, W. H., \& Turner, E. L. 1992, ARA\&A, 30, 499

Fassnacht, C. D., Womble, D. S., Neugebauer, G., Browne, I. W. A., Readhead, A. C. S., Matthews, K., \& Pearson, T. J. 1996, ApJ, 460, L103

Francis, P. J., Hewett, P. C., Foltz, C. B., Chaffee, F. H., Weymann, R. J., \& Morris, S. L. 1991, ApJ, 373, 465

Fukugita, M., Futamase, T., \& Kasai, M. 1990, MNRAS, 246, 24P

Fukugita, M., \& Turner, E. L. 1991, MNRAS, 253, 99

Garrett, M. A., Porcas, R. W., Nair, S., \& Patnaik, A. R. 1996, MNRAS, 279, L7

Gorenstein, M. V., Cohen, N. L., Shapiro, I. I., Rogers, A. E. E., Bonometti, R. J., Falco, E. E., Bartel, N., \& Marcaide, J. M. 1988, ApJ, 334, 42

Jackson, N. J., Nair, S., \& Browne, I. W. A. 1997, in Observational Cosmology with the New Radio Surveys, ed. M. Bremer, N. Jackson, \& I. Perez-Fournon (Dordrecht: Kluwer), 315

Keeton, C. R., Kochanek, C. S., \& Falco, E. E. 1998, ApJ, 509, 561

Kennicutt, R. C., Jr. 1992, ApJS, 79, 255

Kochanek, C. S. 1991, ApJ, 373, 354

Kovner, I., \& Paczyński, B. 1988, ApJ, 335, L9

Kundić, T., Cohen, J. G., Blandford, R. D., \& Lubin, L. M. 1997a, AJ, 114, 507

Kundić, T., Hogg, D. W., Blandford, R. D., Cohen, J. G., Lubin, L. M., \& Larkin, J. E. 1997b, AJ, 114, 2276
Lawrence, C. R., Schneider, D. P., Schmidt, M., Bennett, C. L., Hewitt, J. N., Burke, B. F., Turner, E. L., \& Gunn, J. E. 1984, Science, 223, 46 Leitch, E. M. 1998, Ph.D. thesis, Caltech

Mao, S., \& Schneider, P. 1998, MNRAS, 295, 587

Matthews, K., \& Soifer, B. T. 1994, in Infrared Astronomy with Arrays, ed. I. McLean (Dordrecht: Kluwer), 239

Myers, S. T., et al. 1995, ApJ, 447, L5

. 1999, in preparation

Oke, J. B. 1990, AJ, 99, 1621

Oke, J. B., et al. 1995, PASP, 107, 375

Patnaik, A. R., Browne, I. W. A., Walsh, D., Chaffee, F. H., \& Foltz, C. B. 1992, MNRAS, 259, 1P

Poggianti, B. M. 1997, A\&AS, 122, 399

Postman, M., Lubin, L. M., \& Oke, J. B. 1998, AJ, 116, 560

Press, W. H., Teukolsky, S. A., Vetterling, W. T., \& Flannery, B. P. 1992, Numerical Recipes in C (New York: Cambridge Univ. Press)

Refsdal, S. 1964, MNRAS, 128, 307

Rubin, V. C., Burstein, D., Ford, W. K., Jr., \& Thonnard, N. 1985, ApJ, 289,81

Shepherd, M. C., Pearson, T. J., \& Taylor, G. B. 1994, BAAS, 26, 987

Stetson, P. 1987, PASP, 99, 191

Tonry, J. 1998, AJ, 115, 1

Turner, E. L. 1990, ApJ, 242, L135

Turner, E. L., Ostriker, J. P., \& Gott, J. R. 1984, ApJ, 284, 1

Vogt, N. P., Forbes, D. A., Phillips, A. C., Gronwall, C., Faber, S. M., Illingworth, G. D., \& Koo, D. C. 1996, ApJ, 465, L15 